\title{
Are Younger Patients Undergoing THA Appropriately Characterized as Active?
}

\author{
James A. Keeney MD, Ryan M. Nunley MD, \\ Geneva R. Baca BA, John C. Clohisy MD
}

Received: 8 May 2014/ Accepted: 10 September 2014/Published online: 23 September 2014

(C) The Association of Bone and Joint Surgeons (B) 2014

\begin{abstract}
Background Surgeons perform THA to address a variety of conditions in younger patients, including osteoarthritis (OA), osteonecrosis, inflammatory arthritis, and congenital deformities. Younger patients aged 50 years or younger have been characterized as active in the literature, but a direct relationship between age and activity level has not been well substantiated. Younger patients with OA may engage in higher activity levels; however, associated medical conditions in patients with other surgical indications may not support a generalization that age is a surrogate for activity level. We recently evaluated these issues in younger patients undergoing total knee arthroplasty (TKA) and noted that the majority would not be considered active. Given this observation, we considered whether younger patients undergoing THA are characterized by high activity levels, which is relevant to understanding the long-term risk of wear-related failures.
\end{abstract}

Each author certifies that he or she, or a member of his or her immediate family, has no funding or commercial associations (eg, consultancies, stock ownership, equity interest, patent/licensing arrangements, etc) that might pose a conflict of interest in connection with the submitted article.

All ICMJE Conflict of Interest Forms for authors and Clinical Orthopaedics and Related Research ${ }^{\circledR}$ editors and board members are on file with the publication and can be viewed on request.

Each author certifies that his or her institution approved the human protocol for this investigation, that all investigations were conducted in conformity with ethical principles of research, and that informed consent for participation in the study was obtained.

J. A. Keeney (凹), R. M. Nunley, G. R. Baca, J. C. Clohisy Department of Orthopaedic Surgery, Washington University School of Medicine, 660 South Euclid, CB 8233, St Louis, MO 63110, USA

e-mail: keeneyj@wudosis.wustl.edu
Questions/purposes (1) Do demographic features of younger patients undergoing THA support high activity expectations? (2) Do preoperative or postoperative functional activity measures support projections that younger patients are active after THA?

Methods We retrospectively compared demographic characteristics and functional activity profiles (as determined by preoperative and postoperative UCLA activity scores, Harris hip scores [HHS], and SF-12 and WOMAC physical function subscores) of 704 patients who had undergone THA and were aged younger than 50 years (822 hips) with those of 484 patients (516 hips) aged between 65 and 75 years, who had undergone THA, with a minimum followup at 1 year after surgery (range, 12-160 months). Results Compared with patients aged 65 to 75 years, younger patients undergoing THA were more often men (51\%, 95\% confidence interval [CI], 48.8\%-53.2\% versus $40 \%, 95 \%$ CI, 37.1\%-42.9\% women; p < 0.01 ) or had undergone surgery for osteonecrosis (29\% versus $4 \%$; $95 \%$ CI, 2.8\%-5.2\%; p < 0.001). Postoperative HHS, SF-12, and WOMAC scores were not appreciably different between the two patient groups. Compared with older patients, younger patients with OA had higher preoperative $(5.0 \pm 2.5$ versus $3.9 \pm 2.0, \mathrm{p}<0.001)$ and postoperative UCLA activity scores $(6.8 \pm 2.1$ versus $5.3 \pm 1.9$, $\mathrm{p}<0.001$ ). Younger patients with diagnoses other than OA had slightly higher mean postoperative UCLA activity scores than older patients $(6.0 \pm 2.3$ versus $5.3 \pm 1.9$; $\mathrm{p}<0.001)$. Two hundred fifty-nine of 704 younger patients undergoing THA (37\%; 95\% CI, 34.9\%-39.1\%) returned to impact activity compared with 75 of 484 older patients undergoing THA $(15.5 \%$; 95\% CI, 13.3\%-17.7\%) $(\mathrm{p}<0.001)$.

Conclusions Younger patients with $\mathrm{OA}$ are likely to return to high levels of activity after surgery, which may 
impact long-term wear-related implant survivorship. High activity levels are less common among younger patients with diagnoses other than OA. Age is not the ideal surrogate for activity level in patients considering THA; instead, specific activity-level measures should be used when discussing patient expectations pertaining to postoperative activity levels after arthroplasty.

Level of Evidence Level III, therapeutic study. See Guidelines for Authors for a complete description of levels of evidence.

\section{Introduction}

THA is performed for more than 200,000 patients a year in the United States, including approximately 50,000 procedures for patients aged younger than 55 years old who represent the fastest growing subpopulation undergoing THA [17]. Previously published studies focusing on THA in younger aged patients have reported successful pain relief, functional improvement, and implant survivorship with a variety of implant designs and bearing surfaces [9, $10,12,15-17,22,23,33]$. Although revision rates for THA have been reported more frequently among younger patients undergoing THA, it is difficult to determine whether the higher failure rate reflects demographic considerations for this group or failures of older implant systems or surgical techniques in this patient group $[1,8$, 12, 24-26]. Among studies with well-performing contemporary cementless implants, wear of conventional polyethylene has been identified as the major cause of revision surgery or impending concern for younger patients who have undergone THA [6, 15, 16, 32]. More recently, Babovic and Trousdale [3] reported low wear rates of crosslinked polyethylene among patients aged 50 years or younger but longer followup is still needed to define longevity. Although a variety of diagnoses can result in an indication for THA, patients $\leq 50$ years old have generally been characterized as "active" [3, 5, 15, 16, 23, 33].

Wear-related failures after THA have been associated with higher activity levels and previous characterization of younger patients would suggest a higher risk for long-term, wear-related failure because of their younger age at the time of surgery [27]. However, the majority of studies reporting on outcomes of THA in this patient cohort have not specifically defined their activity levels. Desalotta et al. [6] reported high postoperative activity levels among younger patients who have undergone THA from a telephone survey of 62 patients, but this is a small case series that may not accurately represent this cohort. We previously noted that younger patients undergoing TKA are not uniformly active [13]. Defining demographic characteristics of younger patients undergoing THA and subgroup activity levels are important for targeting implant surveillance as resources for patient followup may become constrained by projected increases in primary and revision THA volume.

Therefore, we assessed the demographic characteristics and activity level profiles (as determined by UCLA activity score, functional subscores of SF-12 and WOMAC instruments, and Harris hip score [HHS]) before and after THA in younger patients (aged $\leq 50$ years) and older patients (aged 65-75 years) who underwent THA at a large, urban teaching hospital. The comparison older group age represents the majority of age-defined patients treated with THA [14, 17, 19]. The purposes of our study were to assess (1) whether demographic features of younger patients support high activity expectations after THA; and (2) whether preoperative or postoperative functional activity measures support characterizations of younger patients undergoing THA as active.

\section{Patients and Methods}

After obtaining institutional review board approval, we retrospectively assessed institutional joint repository data for all patients who had THA performed between January 2000 and December 2009 and had returned for followup at a minimum of 12 months after their THA. Our review identified 704 patients aged 50 years or younger $(822$ THAs) with a mean followup of 52 months (range, 12136 months) and 484 patients between the ages of 65 and 75 years (516 THAs) with a mean followup of 35 months (range, 12-156 months). The older patient age range had previously been used in a parallel study assessing younger TKA patient activity [13]. Patients who had undergone revision surgery during the study interval were excluded from assessment. During the observation period, 1259 THAs had been performed for 1089 patients $\leq 50$ years of age and 980 THAs had been accomplished for 882 patients who were between 65 and 75 years at the time of their surgery. Therefore, the study population represented $65 \%$ of younger patients undergoing THA and 55\% of older patients undergoing THA who had the procedure accomplished during the study period. The $10 \%$ difference in patient followup between groups was statistically significant $(\mathrm{p}<0.001)$ but consistent with previously published followup data from our institution [4]. The greater proportion of younger patients in our institution compared with a registry reporting reflects practices that engaged patients for both hip preservation and hip resurfacing procedures during the intervals of study. Surgeons at our institution independently select indications for THA, but generally, indications include the presence of pain, advanced radiographic disease, and failure of appropriate nonoperative treatment measures. Before 2010, patients at 
our institution were not excluded from surgical intervention on the basis of body mass index (BMI), glycated hemoglobin level, or other potentially modifiable parameters. All reconstructions were accomplished using a cementless acetabular component placed with manual targeting methods and augmented with screw fixation. A cemented stem was used in 201 of 516 hips (39\%) in the older patient group and in seven of 822 hips $(0.7 \%)$ in the younger THA patient group $(\mathrm{p}<0.001)$.

Demographic information, including age, sex, BMI, and surgical diagnosis, was obtained from our institutional data repository. Patient BMI was categorized using the World Health Organization classification for obesity: underweight $\left(<18.5 \mathrm{~kg} / \mathrm{m}^{2}\right)$, normal weight $\left(18.5-25.0 \mathrm{~kg} / \mathrm{m}^{2}\right)$, overweight $\left(25.1-30.0 \mathrm{~kg} / \mathrm{m}^{2}\right)$, Class I obesity $\left(30.1-35.0 \mathrm{~kg} / \mathrm{m}^{2}\right)$, Class II obesity (35.1-40.0 kg/m ${ }^{2}$ ), and Class III obesity $\left(>40 \mathrm{~kg} / \mathrm{m}^{2}\right)$ [34]. Preoperative activity level was determined from patient self-assessment surveys, which included the UCLA activity scale [2]. Functional measures were extracted from the physical function subscore components of WOMAC and SF-12 instruments and from the HHS. All preoperative assessments were obtained within 6 months before the patient undergoing THA. Postoperative UCLA activity scores, SF-12, WOMAC, and HHS were obtained at elective followup evaluations, which occurred at a minimum of 12 months after the surgical procedure (range, 12-156 months). When multiple followup encounters were identified for an individual patient, the latest available followup was selected. To create a categoric distribution of activity, the UCLA activity scale was subclassified into different levels: sedentary (1-2), mildly active (3-4), moderately active (5-7), and highly active (8-10). Patients in both younger and older THA age groups were stratified by sex and diagnosis to assess their activity levels before and after surgery. Statistical analysis was accomplished using a two-tailed t-test for continuous variables (age, BMI, UCLA score, SF-12 mental health and physical function subscores, WOMAC physical function subscore, and HHS) and a two-tailed Fisher's exact test for categorical variables (sex, surgical diagnosis, UCLA activity category, BMI category).

\section{Results}

Comparing Demographics Between Younger and Older Patients

Osteonecrosis was more common in the younger population and osteoarthritis (OA) represented a larger proportion of the older patient population in this study (Table 1). The predominant indications for THA for patients $\leq 50$ years old (822 hips) were OA 517 hips (63\%; 95\% confidence interval [CI], 61\%-65\%) and osteonecrosis 242 hips (31\%; 95\% CI, 29.2\%-32.8\%). Posttraumatic arthritis in 25 hips (3\%) and inflammatory arthropathy in 14 hips (2\%) comprised other discrete minority groups. Among patients with $\mathrm{OA}$ as their indication for surgery, 180 hips $(22 \%$; $95 \% \mathrm{CI}$, 20.3\%-23.7\%) had identifiable acetabular dysplasia, 41 hips (5\%; 95\% CI, 4.1\%-5.9\%) had Perthes deformity of the femoral head, and 33 hips (4\%; 95\% CI, 3.2\%-4.8\%) had a prior slipped capital femoral epiphysis. Among patients between the ages of 65 and 75 years (516 hips), the primary indication for THA was OA in 466 hips $(90 \%$; 95\% CI, 88.4\%-91.6\%). Osteonecrosis was less common than in younger patients and present in 21 hips $(4 \%$; $95 \%$ CI, $2.8 \%-5.2 \%) \quad(\mathrm{p}<0.001)$. Posttraumatic arthritis affected 11 hips (2\%) and inflammatory arthritis affected four hips (1\%). Women were slightly older than men in the older THA patient group $(69.6 \pm 3.1$ years versus men, $69.1 \pm 3.0$ years; $p=0.04)$, whereas men were older than women in the younger THA patient group $(40.2 \pm 9.3$ years versus $37.7 \pm 10.6$ years; $p<0.001)$. BMI was not different between younger and older patients $\left(29.1 \pm 6.8\right.$ versus $\left.29.0 \pm 5.6 \mathrm{~kg} / \mathrm{m}^{2} ; \mathrm{p}=0.62\right)$ and was higher comparing younger and older men who underwent THA $\left(29.8 \pm 6.2\right.$ versus $\left.28.8 \pm 4.5 \mathrm{~kg} / \mathrm{m}^{2} ; \mathrm{p}=0.05\right)$. Although a similar proportion of patients were obese

Table 1. Demographic comparisons of younger ( $\leq 50$ years) and older ( $65-75$ years) patients undergoing THA

\begin{tabular}{|c|c|c|c|c|c|c|c|}
\hline Patient group & $\begin{array}{l}\text { Age } \\
\text { (years) }\end{array}$ & $\begin{array}{l}\text { Sex } \\
\text { (female:male) }\end{array}$ & Race & Diagnosis & $\begin{array}{l}\text { Side } \\
\text { (left:right) }\end{array}$ & $\begin{array}{l}\text { BMI } \\
\left(\mathrm{kg} / \mathrm{m}^{2}\right)^{*}\end{array}$ & $\begin{array}{l}\text { Preoperative } \\
\text { HHS }^{*}\end{array}$ \\
\hline $\begin{array}{l}\text { Older patients with greater } \\
\text { than } 12 \text { months } \\
\text { followup }(\mathrm{n}=516)\end{array}$ & 69.4 & $\begin{array}{l}308: 208 ; \\
\quad \text { female, } \\
59.7 \% ; \\
\text { male, } 40.3 \%\end{array}$ & $\begin{array}{l}\text { White, } 84.9 \% \text {; } \\
\text { black, } 14.5 \% \text {; } \\
\text { Asian/Hispanic, } \\
0.6 \%\end{array}$ & $\begin{array}{l}\text { OA, } 90.3 \% \text {; AVN, } \\
\text { 4.1\%; PT, } 2.1 \% \text {; RA, } \\
1.4 \% \text {; other, } 2.1 \%\end{array}$ & $\begin{array}{l}236: 280 ; \text { left, } \\
\quad 45.7 \% \text {; } \\
\text { right, } \\
54.3 \%\end{array}$ & $29.0 \pm 5.5$ & $\begin{array}{l}41.9 \pm 14.6 \\
\quad(\text { range } \\
4.7-90.7)\end{array}$ \\
\hline $\begin{array}{l}\text { Younger patients with } \\
\text { greater than } 12 \text { months } \\
\text { followup }(\mathrm{n}=822)\end{array}$ & 39.0 & $\begin{array}{l}\text { 402: } 420 ; \\
\text { female, } \\
48.9 \% ; \\
\text { male, } 51.1 \%\end{array}$ & $\begin{array}{l}\text { White, } 83.1 \% \text {; } \\
\text { black, } 15.6 \% \text {; } \\
\text { Asian/Hispanic, } \\
1.3 \%\end{array}$ & $\begin{array}{l}\text { OA, } 62.9 \% \text {; AVN, } \\
\text { 29.4\%; PT, } 3.0 \% \text {; } \\
\text { RA, } 1.7 \% \text {; other } \\
3.0 \%\end{array}$ & $\begin{array}{l}\text { 404:418; left, } \\
\text { 49.2\%; } \\
\text { right, } \\
50.8 \%\end{array}$ & $29.1 \pm 6.8$ & $\begin{array}{l}42.6 \pm 14.4 \\
\quad(\text { range }, \\
10.7-95.4)\end{array}$ \\
\hline
\end{tabular}

*Values are mean \pm SD; BMI = body mass index; HHS = Harris hip score; OA = osteoarthritis; AVN = avascular necrosis; PT = posttraumatic arthritis; $\mathrm{RA}=$ rheumatoid arthritis. 
$\left(\mathrm{BMI}>30 \mathrm{~kg} / \mathrm{m}^{2}\right)$ in both younger and older THA groups (40.6\%, 95\% CI, 38.4\%-42.8\% versus $42.3 \%$, 95\% CI, $39.3 \%-45.3 \%$; $\mathrm{p}=0.59)$, more younger patients undergoing THA had either Class II or III obesity $(17 \%, 95 \% \mathrm{CI}$, $15.3 \%-18.7 \%$ versus $11 \%, 95 \%$ CI, 9.1\%-12.9\%; $\mathrm{p}=0.003$ ). During our study period, bilateral THA was performed twice as often in the younger THA patient group $(14 \%, 95 \% \mathrm{CI}, 12.5 \%-15.5 \%$ versus $6 \%, 95 \% \mathrm{CI}, 4.4 \%-$ $7.6 \% ; \mathrm{p}<0.001)$.

\section{Preoperative Activity Level and Function}

Younger patients who underwent THA had modestly higher mean preoperative UCLA activity scores than older patients $(4.5 \pm 2.4$ versus $3.8 \pm 2.0, \mathrm{p}<0.001)$. Higher preoperative UCLA scores were also noted for younger male patients $(4.7 \pm 2.6$ versus $3.9 \pm 2.3$; p $<0.001)$ and younger female patients $(4.2 \pm 2.1$ versus $3.8 \pm 1.7$; $\mathrm{p}=0.01)$. All preoperative activity scores were also greater for men than women patients (Table 2). Mean preoperative HHS was modestly higher in younger male than younger female patients $(50.2 \pm 14.2$ versus $43.7 \pm 15.1 ; \mathrm{p}=0.01)$. Younger male patients with a diagnosis of OA had higher mean preoperative UCLA activity scores $(5.4 \pm 2.6$ versus $4.0 \pm 2.2 ; \mathrm{p}<0.001)$, HHS $(53.2 \pm 12.5$ versus $47.7 \pm 14.1 ; \mathrm{p}<0.01)$, and WOMAC physical function scores $(47.1 \pm 18.2$ versus $43.7 \pm 18.7 ; \mathrm{p}=0.05$ ) than younger men who underwent
THA with a diagnosis other than OA. Younger female patients had a higher preoperative UCLA activity score than older female patients $(4.2 \pm 2.1$ versus $3.8 \pm 1.7$; $\mathrm{p}=0.01$ ). Younger female patients with OA had higher SF-12 mental function scores than younger female patients with other diagnoses $(31.2 \pm 9.1$ versus $28.6 \pm 9.4$; $\mathrm{p}=0.05)$. The proportion of younger patients who were moderately or highly active was greater than older patients before surgery $(34 \%, 95 \% \mathrm{CI}, 32 \%-36 \%$ versus $23 \%, 95 \%$ CI, 20.5\%-25.5\%; p =0.001) (Fig. 1). Younger patients with a diagnosis other than OA were less likely to be at least moderately active before surgery than younger patients with OA $(23 \%, 95 \%$ CI, $17.7 \%-27.3 \%$ versus $41 \%$, 95\% CI, 34.7\%-47.3\%; p <0.001), but they were proportionally similar to older patients with OA $(23 \%, 95 \%$ CI, $17.7 \%-27.3 \%$ versus $24 \%$, 95\% CI, $21.5 \%-26.5 \%$; $\mathrm{p}=0.79$ ) (Fig. 2).

Postoperative Activity Level and Function: Younger Patients Compared With Older Patients

Mean postoperative UCLA activity scores $(6.4 \pm 2.2$ versus $5.3 \pm 1.9 ; \mathrm{p}<0.001)$ and SF-12 physical function scores $(44.9 \pm 12.4$ versus $42.4 \pm 11.4 ; \mathrm{p}<0.001)$ were higher in younger patients than older patients who underwent THA, but HHS was lower in younger patients $(83.1 \pm 18.0$ versus $86.8 \pm 18.4 ; \mathrm{p}<0.001)$. The lower HHSs were present in both younger female patients

Table 2. Comparison of younger $(<50$ years $)$ and older $(65-75$ years $)$ patients by sex

\begin{tabular}{lllllc}
\hline Age/sex category & $\begin{array}{l}\text { Body mass } \\
\text { index }\left(\mathrm{kg} / \mathrm{m}^{2}\right)\end{array}$ & $\begin{array}{l}\text { Preoperative UCLA } \\
\text { activity score }\end{array}$ & $\begin{array}{l}\text { Preoperative SF-12 } \\
\text { physical function score }\end{array}$ & $\begin{array}{l}\text { Preoperative } \\
\text { Harris hip score }\end{array}$ & $\begin{array}{l}\text { Preoperative WOMAC } \\
\text { physical function score }\end{array}$ \\
\hline All younger patients & $29.1 \pm 6.8$ & $4.5 \pm 2.4$ & $31.4 \pm 9.4$ & $42.6 \pm 14.4$ & $44.8 \pm 19.7$ \\
All older patients & $29.0 \pm 5.5$ & $3.8 \pm 2.0$ & $30.6 \pm 8.9$ & $41.9 \pm 14.6$ & $44.4 \pm 18.5$ \\
All males & $29.5 \pm 5.7$ & $4.7 \pm 2.5$ & $31.9 \pm 9.3$ & $45.0 \pm 14.5$ & $46.5 \pm 19.0$ \\
All females & $28.7 \pm 6.8$ & $3.8 \pm 1.9$ & $30.4 \pm 9.1$ & $42.7 \pm 14.3$ & $43.1 \pm 19.3$ \\
Younger females & $28.4 \pm 7.4$ & $4.2 \pm 2.1$ & $30.4 \pm 9.4$ & $45.9 \pm 14.5$ & $43.5 \pm 20.4$ \\
Older females & $29.1 \pm 6.1$ & $3.8 \pm 1.7$ & $30.4 \pm 8.7$ & $39.7 \pm 14.1$ & $42.3 \pm 18.0$ \\
Younger males & $29.8 \pm 6.2$ & $4.7 \pm 2.6$ & $32.2 \pm 9.3$ & $50.2 \pm 14.2$ & $46.0 \pm 19.0$ \\
Older males & $28.8 \pm 4.5$ & $3.9 \pm 2.3$ & $30.9 \pm 9.3$ & $43.7 \pm 15.1$ & $47.6 \pm 19.1$ \\
p values & & & & 0.42 & 0.8 \\
$\quad \begin{array}{l}\text { All younger versus all } \\
\text { older patients }\end{array}$ & 0.62 & $<0.001$ & 0.27 & $<.001$ & $<0.01$ \\
$\begin{array}{l}\text { All males versus all females } \\
\text { Younger versus older females }\end{array}$ & 0.03 & $<0.001$ & 0.02 & 0.27 & 0.46 \\
$\quad \begin{array}{l}\text { Younger versus older males } \\
\text { Younger females versus }\end{array}$ & 0.03 & 0.01 & 0.98 & 0.52 & 0.43 \\
$\quad \begin{array}{l}\text { younger males } \\
\text { Older females versus older males }\end{array}$ & 0.86 & $<0.001$ & 0.22 & 0.01 & 0.12 \\
\hline
\end{tabular}

Values are mean \pm SD. 


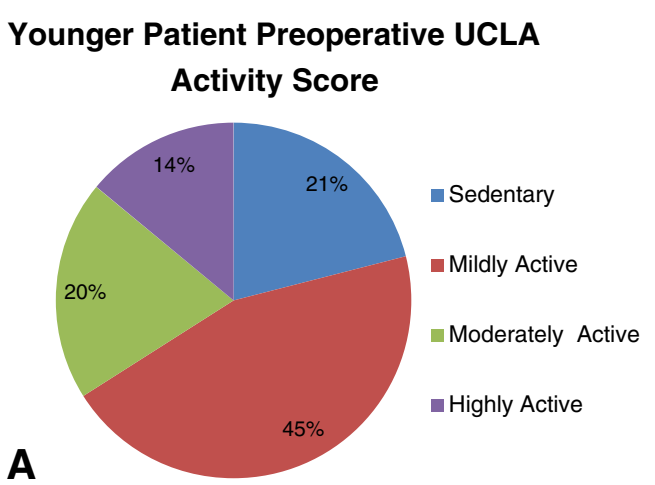

\section{Older Patient Preoperative UCLA Activity Score}

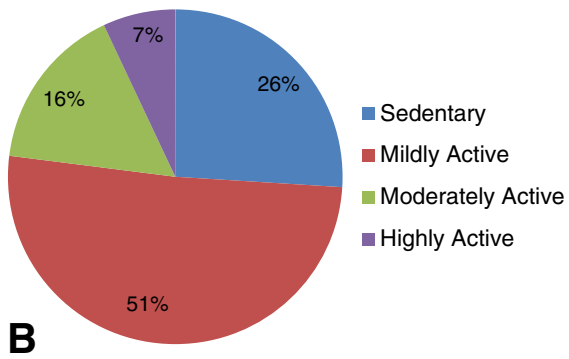

Fig. 1A-B Figures depict the categorical distributions of preoperative UCLA activity scores for (A) younger patients and (B) older patients undergoing THA.
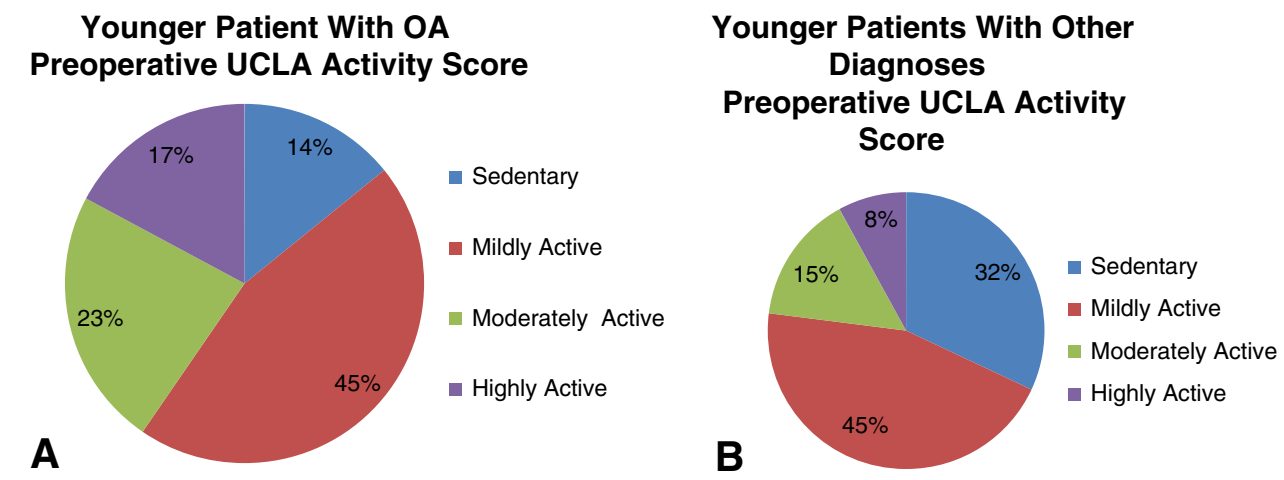

\section{Older Patient With OA \\ Preoperative UCLA Activity Score}

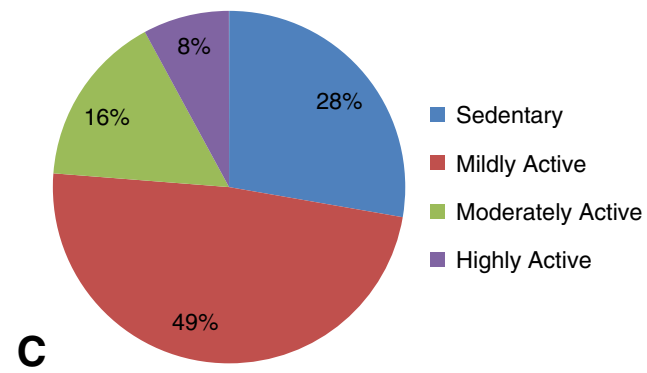

Fig. 2A-C Figures depict the categorical distributions of preoperative UCLA activity scores for (A) younger patients undergoing THA with osteoarthritis (OA), (B) younger patients undergoing THA with other diagnoses, and (C) older patients undergoing THA with a diagnosis of OA.

$(82.1 \pm 18.0$ versus $85.3 \pm 15.2 ; \mathrm{p}=0.02)$ and younger male patients $(84.0 \pm 18.0$ versus $88.9 \pm 13.1 ; \mathrm{p}<0.001)$ (Table 3). Patient sex was associated with postoperative scores with male patients of all ages having higher mean postoperative UCLA activity scores $(6.3 \pm 2.3$ versus $5.5 \pm 2.0 ; \mathrm{p}<0.001)$, SF-12 physical function scores $(45.3 \pm 11.9$ versus $45.3 \pm 11.9 ; \quad \mathrm{p}<0.001), \quad$ HHS $(85.6 \pm 16.7$ versus $83.4 \pm 17.0 ; \mathrm{p}<0.001)$, and WOMAC physical function scores $(82.6 \pm 21.2$ versus $78.7 \pm 23.3 ; \mathrm{p}=0.001)$ compared with female patients.
Younger male patients with OA were more active than younger patients with other diagnoses with higher mean UCLA activity score $(6.5 \pm 2.1$ versus $5.6 \pm 2.3$, $\mathrm{p}<0.001), \mathrm{SF}-12$ physical function subscore $(45.3 \pm 11.0$ versus $43.0 \pm 13.0 ; \mathrm{p}<0.01)$, HHS (85.6 \pm 8.7 versus $83.4 \pm 9.4 ; \mathrm{p}=0.03)$, and WOMAC physical function $(82.6 \pm 19.8$ versus $78.7 \pm 23.3 ; \mathrm{p}=0.001)$. Mean HHS was higher in older male patients with OA than younger male patients with OA $(88.9 \pm 13.1$ versus $84.0 \pm 18.0$; $\mathrm{p}<0.001)$. Younger patients with a diagnosis other than 
Table 3. Comparison of postoperative scores for younger ( $\leq 50$ years) and older ( $65-75$ years) patients

\begin{tabular}{llllll}
\hline Age/sex category & $\begin{array}{l}\text { Followup } \\
\text { (months) }\end{array}$ & $\begin{array}{l}\text { Postoperative } \\
\text { UCLA activity } \\
\text { score }\end{array}$ & $\begin{array}{l}\text { Postoperative SF-12 } \\
\text { physical function } \\
\text { score }\end{array}$ & $\begin{array}{l}\text { Postoperative } \\
\text { Harris hip score }\end{array}$ & $\begin{array}{l}\text { Postoperative WOMAC } \\
\text { physical function score }\end{array}$ \\
\hline All younger patients & $53.3 \pm 38.5$ & $6.4 \pm 2.2$ & $44.9 \pm 12.4$ & $83.1 \pm 18.0$ & $81.3 \pm 22.7$ \\
All older patients & $35.9 \pm 28.4$ & $5.3 \pm 1.9$ & $42.4 \pm 11.4$ & $86.8 \pm 18.4$ & $79.1 \pm 21.9$ \\
All males & $46.1 \pm 36.3$ & $6.3 \pm 2.3$ & $45.3 \pm 11.9$ & $85.6 \pm 16.7$ & $82.6 \pm 21.2$ \\
All females & $46.2 \pm 35.7$ & $5.5 \pm 2.0$ & $43.0 \pm 11.7$ & $83.4 \pm 17.0$ & $78.7 \pm 23.3$ \\
Younger females & $52.7 \pm 38.7$ & $6.0 \pm 2.0$ & $44.0 \pm 12.6$ & $82.1 \pm 18.0$ & $79.8 \pm 23.3$ \\
Older females & $35.5 \pm 28.6$ & $5.0 \pm 1.8$ & $41.2 \pm 11.2$ & $85.3 \pm 15.2$ & $76.8 \pm 10.0$ \\
Younger males & $52.3 \pm 38.4$ & $6.7 \pm 2.3$ & $45.8 \pm 12.1$ & $84.0 \pm 18.0$ & $82.6 \pm 22.1$ \\
Older males & $34.5 \pm 28.1$ & $5.9 \pm 2.0$ & $44.1 \pm 11.4$ & $88.9 \pm 13.1$ & $82.7 \pm 19.0$ \\
Younger versus older patients & $<0.001$ & $<0.001$ & $<0.001$ & $<0.001$ & 0.11 \\
All males versus all females & 0.66 & $<0.001$ & $<0.01$ & 0.03 & 0.001 \\
Younger versus older females & $<0.001$ & $<0.001$ & $<0.01$ & 0.02 & 0.10 \\
Younger versus older males & $<0.001$ & $<0.001$ & 0.14 & $<0.001$ & 0.95 \\
Younger females versus & 0.65 & $<0.001$ & 0.05 & 0.11 & 0.08 \\
$\quad$ younger males & & $<0.001$ & 0.01 & $<0.01$ & $<0.01$ \\
Older females-older males & 0.78 & & &
\end{tabular}

Values are mean \pm SD.

OA had higher mean postoperative activity UCLA score compared with older patients with $\mathrm{OA}(6.0 \pm 2.1$ versus $5.4 \pm 1.9 ; \mathrm{p}<0.001)$, similar values for SF-12 physical function $(45.3 \pm 11.0$ versus $44.7 \pm 11.3 ; \mathrm{p}=0.01)$, and WOMAC physical function subscore $(82.6 \pm 19.8$ versus $84.0 \pm 18.6 ; \mathrm{p}=0.59)$. Compared with older female patients, younger female patients had higher postoperative UCLA activity scores $(6.0 \pm 2.0$ versus $5.0 \pm 1.8$; $\mathrm{p}<0.001)$ and SF-12 physical function subscore $(44.0 \pm 12.6$ versus $41.2 \pm 11.2 ; \mathrm{p}<0.01)$ but a lower HHS (82.1 \pm 18.0 versus $85.3 \pm 15.2$; $\mathrm{p}=0.02$ ). Compared with younger female patients with other diagnoses, younger female patients with OA had higher postoperative SF-12 physical function subscores (45.5 \pm 12.2 versus $40.7 \pm 12.0 ; \mathrm{p}=0.05)$ and there were trends toward higher UCLA activity $(6.2 \pm 1.9$ versus $5.5 \pm 2.2$; $\mathrm{p}=0.07)$ and SF-12 mental health $(52.3 \pm 10.2$ versus $48.0 \pm 9.6 ; \mathrm{p}=0.06$ ). A categorical increase in BMI was associated with decreasing postoperative UCLA activity scores in women patients, but no such association was seen among male patients. Female patients with a BMI less than $30 \mathrm{~kg} / \mathrm{m}^{2}$ had a higher mean postoperative UCLA activity score than female patients with a BMI greater than $35 \mathrm{~kg} / \mathrm{m}^{2}$ $(5.8 \pm 2$ versus $4.7 \pm 1.8 ; \mathrm{p}<0.001)$. In contrast, mean postoperative UCLA activity scores in male patients with a BMI less than $30 \mathrm{~kg} / \mathrm{m}^{2}$ did not differ from mean UCLA activity scores for men with a BMI greater than $35 \mathrm{~kg} / \mathrm{m}^{2}$ $(6.5 \pm 2.2$ versus $6.2 \pm 2.4 ; \mathrm{p}=0.42)$.

After THA, a similar proportion of patients in both the younger (598 of 704 patients [85\%]; 95\% CI, 83.5\%$86.5 \%$ ) and older age groups (411 of 484 patients [85\%];
95\% CI, 82.9\%-87.9\%) experienced an increase in their activity level using the UCLA score. However, younger patients were categorically more likely than older patients to return to impact activities $(37 \%, 95 \%$ CI, 34.9\%-39.1\% versus $15.5 \%, 95 \% \mathrm{CI}, 13.3 \%-17.7 \%$; $\mathrm{p}<0.001)$ and younger patients with $\mathrm{OA}$ were more likely to return to at least moderate activity than older patients with OA $(81 \%$, $95 \% \mathrm{CI}, 79.4 \%-82.6 \%$ versus $61 \%$, 95\% CI, $58.4 \%-$ 63.6\%; p < 0.001) (Fig. 3).

\section{Discussion}

The use of THA has increased substantially over the past two decades, particularly among younger patients [17]. Considerations regarding implant selection, surgical technique, and perioperative patient management are uniquely relevant for younger patients because their life expectancy may exceed the durability of contemporary prosthetic implants. Although several studies have reported improvement in clinical and function scores in younger patients, concerns remain regarding the longevity of bearing surfaces under high demand among the most active younger patients $[6,15,16,33]$. There have been few studies that directly assessed both pre- and postoperative patient activity in patients $\leq 50$ years of age $[6,29,32]$. We recently evaluated these issues in younger patients undergoing TKA $\leq 55$ years and noted that the majority did not have demographic features that supported an expectation for high activity levels. Furthermore, the proportion of patients engaging in high levels of activity after 


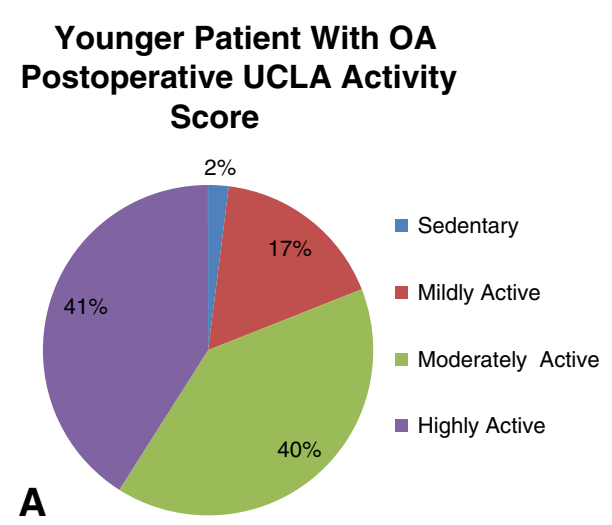

Older Patient With OA Postoperative UCLA Activity Score

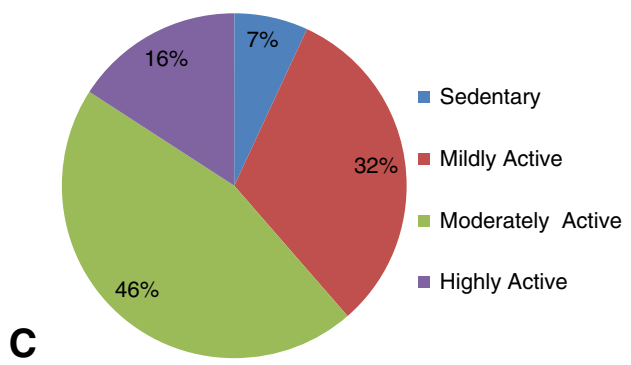

\section{Younger Patient With Other \\ Diagnoses Postoperative UCLA Activity Score}

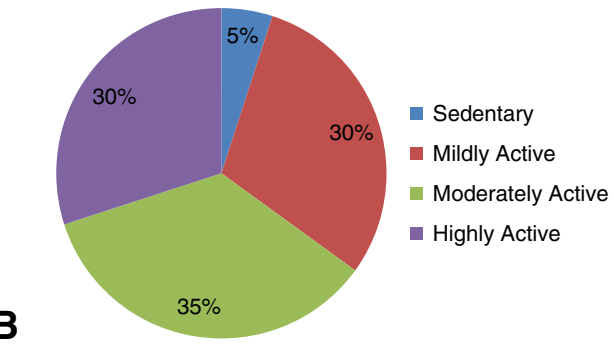

Fig. 3A-C Figures depict the categorical distributions of postoperative UCLA activity scores for (A) younger patients with OA, (B) younger patients with other diagnoses, and (C) older patients with a diagnosis of OA undergoing THAs.

TKA was not substantially different than older patients who were 65 to 75 years of age [11]. It is important to consider patients undergoing THA separately because their demographic features and activity levels have not been previously characterized. Understanding this younger THA patient group is important to define patients at the greatest risk of wear-related implant failure and to target surveillance under anticipated resource constraints for conducting routine joint arthroplasty assessment. We therefore sought to compare (1) demographic characteristics and (2) activity level profiles (as determined by UCLA score, SF-12 and WOMAC physical function subscores, and HHSs) before and after THA in younger (aged younger than 50 years) and older (aged 65-75 years) patients treated at a large, urban teaching hospital. In this study, we found that THA is a successful procedure, resulting in increased functional activity as defined by the UCLA activity score for $85 \%$ of patients in both the younger and older THA groups. Younger patients undergoing THA with OA had the highest postoperative activity levels and higher functional outcome scores than older patients undergoing THA. Most younger patients undergoing THA $(81 \%)$ returned to at least moderate activity, and a substantial proportion (37\%) returned to impact activity or recreational sports. In our previous TKA study, we had noted only $57 \%$ of younger patients undergoing TKA reporting an increase in UCLA activity score, $44 \%$ returning to at least moderate activity, and only $10 \%$ returning to impact activity or recreational sports [13]. The observations of this study support that many younger patients undergoing THA perform at comparatively higher activity levels with the potential for this to impact wear-related implant longevity.

There are several notable limitations of our study. We did not specifically address the severity of concurrent musculoskeletal disease processes that may have impacted activity levels in either age group. We would generally anticipate a higher prevalence of systemic disease among both older patients in general and younger patients with a diagnosis of osteonecrosis or inflammatory arthritis; however, we did not consider such aspects and this could have influenced our study findings. The proportion of patients with bilateral THA was higher among younger patients during the study interval but we were not able to assess for the presence of prior contralateral joint reconstruction among patients or the presence of other musculoskeletal disease processes that may have influenced either preoperative or postoperative activity scores. Use of scores available at the latest followup appointment potentially could reduce functional activity measures and create lower estimates of patient activity. Such differences would be 
expected to be most substantial among older patients who were 30 years older on average than younger patients undergoing THA at the time of surgery. The minimum interval of followup was also shorter than ideal for assessing potential effects related to a minority of implants that might be failing; however, because the purpose of our study was to address functional activity levels rather than implant survivorship, the 12-month minimum followup interval was accepted as an adequate time period for patient functional activity to recover to a predisease or presurgical baseline. The demographic characteristics of patients in this study reflect a tertiary hospital practice that has included hip preservation surgery, which may explain the higher proportion of younger patients undergoing THA noted in this study compared with national registry-based studies $[14,18,19]$. The demographic characteristics are also representative of a US patient population, so activity considerations may not be generalized to other cultures.

Our study used UCLA activity level and postoperative functional subscores as a measure of patient activity instead of direct measurement with a pedometer or stepankle-monitor (SAM) device reported in other studies [27, 29, 30, 32, 35]. However, pedometer data were used to validate the UCLA activity scale for postoperative THA patient assessment that was used in this study [35]. Silva et al. [30] reported higher accuracy of a SAM compared with a pedometer, an average of two million cycles per year for patients undergoing THA, and 63\% higher activity in patients $<60$ years old, but made these observations using only five of 33 patients $(15 \%)<60$ years old. Two studies have specifically documented pedometer for patients undergoing THA $\leq 50$ years, but the information is difficult to interpret because only mean pedometer and ranges of activity levels were reported [29, 32]. Takenaga et al. [32] noted a broad range of pedometer measured activity (77,000-3.26 million cycles/year) in 50 patients (58 hips) and Sechriest et al. [29] also noted a broad range of activity (0.3-2.7 million cycles/year) among 34 patients undergoing THA (41 hips). Although improvements in mean postoperative functional scores were statistically significant as a result of the large number of patients included in this study, these differences are generally smaller than the minimum clinically important differences (MCIDs) that have been reported for THA. Smith et al. [31] reported an MCID range for HHS between 7 and 9 points and an MCID for WOMAC physical function subscore as $12 \%$ of baseline score or $6 \%$ of maximum score (5-6 points). Furthermore, although activity levels may be helpful in defining patients at risk, it is important to note that the majority of failures after THA may not be related to wear. Studies reporting from national registries have identified component loosening, infection, and dislocation as the most common reasons for surgical revision $[11,14]$.
Melvin et al. [21] reported that $24 \%$ of patients underwent surgical revision within 5 years of the index procedure with the most common reasons for revision including aseptic loosening of components, infection, and dislocation. However, early failures are expected to present symptomatically within the first few years after THA and should not influence considerations of targeted surveillance for wear-related failures among the most active younger patients undergoing THA.

The younger THA cohort in our study included a large number of patients with osteonecrosis, similar to the proportion reported by Crowther and Lachiewicz [5]. The younger patients were also more often men, and higher activity levels were noted specifically among male patients with OA. Schmalzried et al. [27] previously associated a higher incidence of polyethylene wear with male patients and increased activity levels among a small cohort of 24 patients. Crowther and Lachiewicz [5] associated increased conventional polyethylene wear rates and periprosthetic osteolysis with higher postoperative HHS in younger patients. Because average HHS was higher in older male and female patients than younger patients in our study-the opposite of other functional activity measures that were higher in younger patients-our results suggest that other measures of functional activity may be more appropriate to measure and report than the HHS when attempting to correlate bearing surface wear with patient activity.

Before surgical intervention, younger patients undergoing THA had higher mean UCLA activity scores than older patients, but other traditional THA outcome scores were not substantially different. After surgery, UCLA activity levels increased for both younger and older patients. Other functional scores had a variable response, but older patients had higher mean HHSs, indicating a limited benefit in the use of using this measure to assess postoperative THA outcomes. Although some measures demonstrated statistically significant differences between younger and older THA patient groups, the differences fell below the thresholds for MCIDs for SF-12, WOMAC, and HHS.

Mean functional activity scores increased for patients in both age groups, for both sexes, and among patients with either OA or other diagnoses. Our study findings suggest that the younger THA cohort-especially those patients with a diagnosis of $\mathrm{OA}$ - is appropriately characterized by a higher postoperative activity profile when compared with patients undergoing THA between ages 65 and 75 years. A substantial number of younger patients with OA $(40 \%)$ and other diagnoses $(30 \%)$ participated in impact sports activities at a mean of 50 months after their surgical procedure, but approximately $40 \%$ of younger patients undergoing THA with a diagnosis other than OA were sedentary or only mildly active. Long-term failure from bearing surface wear and osteolysis may have less impact for this subset of 
younger patients undergoing THA. Babovic and Trousdale [3] have reported minimal wear of highly crosslinked polyethylene bearings for younger patients undergoing THA at a minimum of 10 years after surgery. Long-term assessment of wear among the most active patients will be important to delineate the durability of contemporary THA.

THA has demonstrated effectiveness for lessening pain and improving function for younger patients $[3,5,10,16$, $17,20,22,28]$. In our study, activity level and functional scores increased the most substantially for younger male and female patients with a diagnosis of OA. Younger patients with other diagnoses had more variable activity levels after surgery, but as many as $30 \%$ of them returned to impact activities. Other published studies have supported that increased activity levels are the most predictive of increased rates of bearing surface wear and osteolysis [5, 27]. Our study findings suggest that enhancements in implant designs that are intended to allow greater durability under high activity levels may have an effect for as many as $40 \%$ of all younger patients undergoing THA. Determining the impact of activity on survivorship of prosthetic designs and techniques should be based on measured patient-function levels rather than using age as a surrogate for activity.

\section{References}

1. Aldinger PR, Jung AW, Pritsch M, Breusch S, Thomsen M, Ewerbeck V, Parsch D. Uncemented grit-blasted straight tapered titanium stems in patients younger than fifty-five years of age. Fifteen to twenty-year results. $J$ Bone Joint Surg Am. 2009;91:1432-1439.

2. Amstutz HC, Thomas BJ, Jinnah R, Kim W, Grogan T, Yale C. Treatment of primary osteoarthritis of the hip. A comparison of total joint and surface replacement arthroplasty. J Bone Joint Surg Am. 1984;66:228-241.

3. Babovic N, Trousdale RT. Total hip arthroplasty using highly cross-linked polyethylene in patients younger than 50 years with minimum 10-year follow-up. J Arthroplasty. 2013;28:815-817.

4. Clohisy JC, Kamath GV, Byrd GD, Steger-May K, Wright RW. Patient compliance with clinical follow-up after total joint arthroplasty. J Bone Joint Surg Am. 2008;90:1848-1854.

5. Crowther JD, Lachiewicz PF. Survival and polyethylene wear of porous-coated acetabular components in patients less than fifty years old: results at nine to fourteen years. J Bone Joint Surg Am. 2002;84:729-735.

6. Delasotta LA, Rangavajjula AV, Porat MD, Frank ML, Orozco FR, Ong AC. What are young patients doing after hip reconstruction? J Arthroplasty. 2012;27:1518-1525.

7. Eskelinen A, Remes V, Helenius I, Pulkkinen P, Nevalainen J, Paavolainen P. Total hip arthroplasty for primary osteoarthrosis in younger patients in the Finnish arthroplasty register. 4,661 primary replacements followed for 0-22 years. Acta Orthop. 2005;76:28-41.

8. Eskelinen A, Remes V, Helenius I, Pulkkinen P, Nevalainen J, Paavolainen P. Uncemented total hip arthroplasty for primary osteoarthritis in young patients: a mid-to long-term follow-up study from the Finnish Arthroplasty Register. Acta Orthop. 2006;77:57-70.

9. Ha YC, Koo KH, Jeong ST, Joon Yoo J, Kim YM, Joong Kim H. Cementless alumina-on-alumina total hip arthroplasty in patients younger than 50 years: a 5 -year minimum follow-up study. $J$ Arthroplasty. 2007;22:184-188.

10. Innmann MM, Gotterbarm T, Kretzer JP, Merle C, Ewerbeck V, Weiss S, Aldinger PR, Streit MR. Minimum ten-year results of a 28-mm metal-on-metal bearing in cementless total hip arthroplasty in patients fifty years of age and younger. Int Orthop. 2014;38:929-934.

11. Jameson SS, Mason JM, Baker PN, Jettoo P, Deehan DJ, Reed MR. Factors influencing revision risk following 15740 singlebrand hybrid hip arthroplasties: a cohort study from a National Joint Registry. J Arthroplasty. 2013;28:1152-1159.

12. Keener JD, Callaghan JJ, Goetz DD, Pederson DR, Sullivan PM, Johnston RC. Twenty-five-year results after Charnley total hip arthroplasty in patients less than fifty years old: a concise follow-up of a previous report. J Bone Joint Surg Am. 2003;85:1066-1072.

13. Keeney JA, Nunley RM, Wright RW, Barrack RL, Clohisy JC. Are younger patients undergoing TKAs appropriately characterized as active? Clin Orthop Relat Res. 2014;472:1210-1216.

14. Khatod M, Cafri G, Namba RS, Inacio MC, Paxton EW. Risk factors for total hip arthroplasty aseptic revision. J Arthroplasty. 2014;29:1412-1417.

15. Kim YH, Kook HK, Kim JS. Total hip replacement with a cementless acetabular component and a cemented femoral component in patients younger than fifty years of age. J Bone Joint Surg Am. 2002;84:770-774.

16. Kim YH, Oh SH, Kim JS. Primary total hip arthroplasty with a second-generation cementless total hip prosthesis in patients younger than fifty years of age. J Bone Joint Surg Am. 2003;85:109-114.

17. Kurtz SM, Lau E, Ong K, Zhao K, Kelly M, Bozic KJ. Future young patient demand for primary and revision joint replacement: national projections from 2010 to 2030. Clin Orthop Relat Res. 2009;467:2606-2612.

18. Lindgren JV, Wretenberg P, Kärrholm J, Garellick G, Rolfson O. Patient-reported outcome is influenced by surgical approach in total hip replacement: a study of the Swedish Hip Arthroplasty Register including 42,233 patients. Bone Joint J. 2014;96: 590-596.

19. Mäkelä KT, Matilainen M, Pulkkinen P, Fenstad AM, Havelin LI, Engesaeter L, Furnes O, Overgaard S, Pedersen AB, Kärrholm J, Malchau H, Garellick G, Ranstam J, Eskelinen A. Countrywise results of total hip replacement. An analysis of 438,733 hips based on the Nordic Arthroplasty Register Association database. Acta Orthop. 2014;85:107-116.

20. Matharu GS, McBryde CW, Pynsent WB, Pynsent PB, Treacy RB. The outcome of the Birmingham Hip Resurfacing in patients aged $<50$ years up to 14 years post-operatively. Bone Joint J. 2013;95:1172-1177.

21. Melvin JS, Karthikeyan T, Cope R, Fehring TK. Early failures in total hip arthroplasty-a changing paradigm. J Arthroplasty. 2014;29:1284-1288.

22. Migaud H, Jobin A, Chantelot C, Giraud F, Laffargue P, Duquennoy A. Cementless metal-on-metal hip arthroplasty in patients less than 50 years of age: comparison with a matched control group using ceramic-on-polyethylene after a minimum 5-year follow-up. J Arthroplasty. 2004;19(Suppl 3):23-28.

23. Moyer JA, Metz CM, Callaghan JJ, Hennessy DW, Liu SS. Durability of second-generation extensively porous-coated stems in patients age 50 and younger. Clin Orthop Relat Res. 2010;468:448-453.

24. Pedersen AB, Mehnert F, Havelin LI, Furnes O, Herberts P, Kärrholm J, Garellick G, Mäkela K, Eskelinen A, Overgaard S. 
Association between fixation technique and revision risk in total hip arthroplasty patients younger than 55 years of age. Results from the Nordic Arthroplasty Register Association. Osteoarthritis Cartilage. 2014;22:659-667.

25. Prokopetz JJ, Losina E, Bliss RL, Wright J, Baron JA, Katz JN. Risk factors for revision of primary total hip arthroplasty: a systematic review. BMC Musculoskelet Disord. 2012;13:251.

26. Santaguida PL, Hawker GA, Hudak PL, Glazier R, Mahomed NN, Kreder HJ, Coyte PC, Wright JG. Patient characteristics affecting the prognosis of total hip and knee joint arthroplasty: a systematic review. Can J Surg. 2008;51:428-436.

27. Schmalzried TP, Shepherd EF, Dorey FJ, Jackson WO, dela Rosa M, Fa'vae F, McKellop HA, McClung CD, Martell J, Moreland JR, Amstutz HC. The John Charnley Award. Wear is a function of use, not time. Clin Orthop Relat Res. 2000;381:36-46.

28. Schmitz MW, Timmer C, Rijnen WH, Gardeniers JW, Schreurs BW. Clinical and radiological outcome of the cemented Contemporary acetabular component in patients $<50$ years of age. Bone Joint J. 2013;95:1617-1625.

29. Sechriest VF 2nd, Kyle RF, Marek DJ, Spates JD, Saleh KJ, Kuskowski M. Activity level in young patients with primary total hip arthroplasty: a 5-year minimum follow-up. J Arthroplasty. 2007;22:39-47.
30. Silva M, Shepherd EF, Jackson WO, Dorey FJ, Schmalzried TP. Average patient walking activity approaches 2 million cycles per year: pedometers under-record walking activity. $J$ Arthroplasty. 2002;17:693-697.

31. Smith MV, Klein SE, Clohisy JC, Baca GR, Brophy RH, Wright RW. Lower extremity-specific measures of disability and outcomes in orthopaedic surgery. $J$ Bone Joint Surg Am. 2012;94:468-477.

32. Takenaga RK, Callaghan JJ, Bedard NA, Liu SS, Gao Y. Which functional assessments predict long-term wear after total hip arthroplasty? Clin Orthop Relat Res. 2013;471:2586-2594.

33. Takenaga RK, Callaghan JJ, Bedard NA, Liu SS, Klaassen AL, Pedersen DR. Cementless total hip arthroplasty in patients fifty years of age or younger: a minimum ten-year follow-up. J Bone Joint Surg Am. 2012;94:2153-2159.

34. World Health Organization. Preventing and Managing the Global Obesity Epidemic: Report of a WHO Consultation. Geneva, Switzerland: WHO; 2000:8.

35. Zahiri CA, Schmalzried TP, Szuszczewicz ES, Amstutz HC. Assessing activity in joint replacement patients. J Arthroplasty. 1998;13:890-895. 